\title{
EFEITO DE FUNGOS MICORRÍZICOS ARBUSCULARES ASSOCIADOS A COMPOSTOS FENÓLICOS, NO CRESCIMENTO DE MUDAS DE MAMOEIRO1
}

\author{
MARCO ANTONIO MARTINS ${ }^{2}$, GEISA FONSECA DE GONÇALVES ${ }^{3}$ e ANA CRISTINA FERMINO SOARES ${ }^{4}$
}

\begin{abstract}
RESUMO - Este trabalho foi conduzido em casa de vegetação com objetivo de avaliar os efeitos de diferentes espécies de fungos micorrízicos arbusculares (FMAs) associados a compostos fenólicos (rutina e quercetina), sobre o crescimento e os conteúdos de $\mathrm{P}$ de mudas de mamoeiro, sob duas doses de $\mathrm{P}\left(0 \mathrm{e} 50 \mathrm{mg} \mathrm{kg}^{-1}\right)$. As espécies de fungos utilizadas foram Glomus clarum $(\mathrm{Gc})$, G. macrocarpum $(\mathrm{Gm})$ e uma população nativa de FMAs (Pn) isolada de uma área de plantio de mamão na Estação Experimental da PESAGRO-RIO, em Macaé, RJ. Na dose $0 \mathrm{mg} \mathrm{kg}^{-1}$ de P, a inoculação de Gc aumentou significativamente a produção de matéria seca e os conteúdos de $\mathrm{P}$ da parte aérea, independentemente da adição dos compostos rutina ou quercetina. Entretanto, no caso da espécie Gm, estes aumentos só foram observados no tratamento onde se adicionou rutina, o que indica que este composto aumentou a eficiência desta espécie. Não ocorreu influencia significativa da Pn sobre a produção de matéria seca e os conteúdos de $\mathrm{P}$ da parte aérea. Com o aumento da dose $\mathrm{P}\left(50 \mathrm{mg} \mathrm{kg}^{-1}\right)$, as espécies de FMAs utilizadas não induziram aumento significativo na produção de matéria seca e no conteúdo de $\mathrm{P}$ da parte aérea do mamoeiro.
\end{abstract}

Termos para indexação: micorriza, Glomus, rutina, quercetina, fósforo, matéria seca.

\section{EFFECTS OF ARBUSCULAR MYCORRHIZAL FUNGI ASSOCIATED WITH PHENOLIC COMPOUNDS ON THE GROWTH OF PAPAYA}

\begin{abstract}
This work was carried out under greenhouse conditions to evaluate the effects of different species of arbuscular mycorrhizal fungi (AMF), associated with phenolic compounds (rutin and quercetin) on the growth and $\mathrm{P}$ contents of papaya plants under two doses of $\mathrm{P}\left(0\right.$ and $\left.50 \mathrm{mg} \mathrm{\textrm {kg } ^ { - 1 }}\right)$. The fungi used were Glomus clarum $(\mathrm{Gc})$, G macrocarpum $(\mathrm{Gm})$ and a population of native fungi $(\mathrm{Nf})$, which was isolated from a papaya plantation in the Experimental Station of PESAGRO-RIO, Macaé, RJ, Brazil. At the dose $0 \mathrm{mg} \mathrm{kg}^{-1}$ of $\mathrm{P}$, the inoculation with Gc led to significant increases in dry matter and $\mathrm{P}$ contents of plant shoots, independently of the addition of the compounds rutin or quercetin. However, in the case of $\mathrm{Gm}$, significant increases was only observed in treatments in which rutin was added, indicating that this compound was able to increase the effectiveness of this fungal species. There was no significant effects of $\mathrm{Nf}$ on the dry matter and $\mathrm{P}$ content of plant shoots. Increasing the doses of $\mathrm{P}\left(50 \mathrm{mg} \mathrm{kg}^{-1}\right)$, both AMF species used did not lead to any significant increase in dry matter and $\mathrm{P}$ content of papaya plant shoots.
\end{abstract}

Index terms: mycorrhizae, Glomus, rutin, quercetin, phosphorus, dry matter.

\footnotetext{
${ }^{1}$ Aceito para publicação em 19 de julho de 1999.

${ }^{2}$ Eng. Agrôn., Ph.D., Prof. Associado, Centro de Ciências e Tecnologias Agropecuárias (CCTA), Universidade Estadual do Norte Fluminense (UENF), Av. Alberto Lamego 2.000, CEP 28015-620 Campos dos Goytacazes, RJ. E-mail: marco@uenf.br

${ }^{3}$ Biól., em curso de mestrado, Centro de Biociências e Biotecnologias (CBB), UENF.

${ }^{4}$ Microbiologista, D.Sc., Prof. Titular, Dep. de Fitotecnia, Escola de Agronomia, Universidade Federal da Bahia, CEP 44380-000 Cruz das Almas, BA.
}

\section{INTRODUÇÃO}

A região norte/noroeste do Estado do Rio de Janeiro é caracterizada quase que exclusivamente pela monocultura da cana-de-açúcar, e a busca de alternativas para diversificação agrícola, como a fruticultura, torna-se fator importante para o seu desenvolvimento agrícola. Devido à existência de condições edafoclimáticas semelhantes às das principais regiões produtoras de mamão do país, à proximidade dos grandes centros consumidores do produto, à gran- 
de oferta de mão-de-obra e à reduzida incidência de virose na região, a cultura do mamoeiro pode constituir uma grande alternativa para a diversificação agrícola. Entretanto, o grande entrave para o melhor desenvolvimento da fruticultura é a pouca disponibilidade de mudas selecionadas; existe a necessidade da instalação de um programa de produção de mudas de boa qualidade, para as diversas fruteiras de interesse econômico. Deste modo, a produção de mudas de fruteiras de alta qualidade e de interesse econômico é fator importante para atender este mercado promissor de grande relevância.

A incorporação da inoculação de fungos micorrízicos arbusculares (FMAs), no sistema de produção de mudas micropropagadas ou de sementes selecionadas, tem demonstrado grande potencial como alternativa biológica para o desenvolvimento de um cultivo racional e eficiente de mudas de diversas fruteiras (Schubert et al., 1990; Jaizme-Vega \& Azcón, 1991, 1995). Os FMAs proporcionam um melhor desenvolvimento das mudas, dada a maior absorção de certos nutrientes, principalmente do fósforo, melhor resistência ao estresse hídrico, melhor aclimatação de mudas micropropagadas, maior resistência ao transplante e tolerância a patógenos (Jaizme-Vega \& Azcón, 1991, 1995). O maior desenvolvimento das mudas colonizadas por FMA pode, não somente reduzir o tempo de permanência das mudas no viveiro, reduzindo os custos de insumos $\mathrm{e}$ mão-de-obra, mas também, por causa do maior vigor e sobrevivência das mudas após transplantio para o campo, reduzir os custos adicionais com o replantio.

Os FMAs são de ocorrência natural no solo e são encontrados sob quase todas condições edafoclimáticas. Entretanto, muitos desses fungos nativos possuem baixa eficiência micorrízica, ou seja, não são eficientes em promover o crescimento da planta hospedeira. Assim, para a integração da inoculação de FMAs, em um programa de produção de mudas de frutíferas, é necessário fazer um estudo sobre a ocorrência de FMAs nativos do solo, e avaliar a eficiência destes e de espécies exóticas que proporcionem uma simbiose efetiva entre a espécie frutífera e a espécie fúngica.

Diversos estudos têm demonstrado que compostos fenólicos, especialmente do grupo dos flavonóides, os quais são geralmente produzidos pelas raízes como resposta de defesa à presença de patógenos, tendem a estimular o desenvolvimento de diversas espécies de FMAs (Elias \& Safir, 1987; Tsai \& Phillips, 1991; Bécard et al., 1992; Baptista \& Siqueira, 1994), aumentando, assim, o benefício dos FMAs no desenvolvimento das plantas hospedeiras.

Este trabalho teve como objetivo avaliar o desenvolvimento de mudas de mamoeiro (cultivar Improved Sunrise Solo Line 72/12), que receberam inoculação de diferentes espécies de FMA, e avaliar o efeito dos compostos fenólicos rutina e quercetina sobre a colonização dos FMAs.

\section{MATERIAL E MÉTODOS}

\section{Preparo do inóculo de FMA}

O substrato utilizado para o preparo do inóculo consistiu em uma mistura de solo e areia na proporção de 1:2 (v/v). O substrato foi esterilizado em autoclave por três vezes, a uma temperatura de $121^{\circ} \mathrm{C}$, por uma hora. $\mathrm{O}$ substrato foi colocado em vasos de cultivo com $5 \mathrm{dm}^{3} \mathrm{de}$ capacidade e infectado com uma mistura de solo contendo esporos e raízes colonizadas ( $50 \mathrm{~g}$ de inóculo) com o fungo de cada espécie a ser estudada: Glomus clarum, G. macrocarpum, e população nativa. O inóculo nativo foi coletado em uma área de plantio de mamão na Estação Experimental da PESAGRO-RIO, em Macaé, RJ. Foram identificadas as seguintes espécies na população de fungos nativos (Pn): G. clarum, G. spurcum, Scutellospora fulgida, G. macrocarpum, G. invermaium, Entrophospora colombiana, S. pellucida, Acaulospora appendiculata e $S$. heterogama. G. clarum e G. spurcum foram as espécies predominantes.

Para a multiplicação do inóculo, foram semeadas seis sementes de Brachiaria bryzantha, que tiveram a superfície esterilizada com solução de $0,5 \%$ de hipoclorito de sódio, durante 15 minutos. Após a embebição, as sementes foram lavadas com água esterilizada, por quatro vezes consecutivas. Os vasos foram mantidos em casa de vegetação por um período de quatro a seis meses para a multiplicação dos fungos, os quais foram utilizados como inóculo.

\section{Preparo do substrato para a produção das mudas}

O substrato para as mudas foi composto por uma mistura de solo peneirado em malha de $2 \mathrm{~mm}$ e areia na proporção de $1: 2(\mathrm{v} / \mathrm{v})$. A areia foi lavada e misturada com o 
solo, e, em seguida, o substrato foi desinfestado com brometo de metila, de acordo com as recomendações do fabricante, para eliminação de possíveis FMAs nativos do substrato. A análise química do substrato mostrou os seguintes resultados: $\mathrm{pH}$ (água), 5,6; $\mathrm{P}, 6 \mathrm{mg} \mathrm{kg}^{-1}$; $\mathrm{K}, 0,87 \mathrm{mmol}_{\mathrm{c}} \mathrm{kg}^{-1}$; Ca, 3,2 $\mathrm{mmol}_{\mathrm{c}} \mathrm{kg}^{-1}$; $\mathrm{Mg}, 2,2$ mmol $_{\mathrm{c}} \mathrm{kg}^{-1}$, $\mathrm{Al}, 0,2 \mathrm{mmol}_{\mathrm{c}} \mathrm{kg}^{-1}$; H total, 18 mmol $_{\mathrm{c}} \mathrm{kg}^{-1} ; \mathrm{Na}, 0,8$ mmol $_{\mathrm{c}} \mathrm{kg}^{-1}$; C, $6,8 \mathrm{~g} \mathrm{~kg}^{-1} ; \mathrm{N}$ total, $3,1 \mathrm{~g} \mathrm{~kg}^{-1}$.

Ao substrato adicionaram-se duas doses de $\mathrm{P}(0$ e $50 \mathrm{mg} \mathrm{kg}^{-1}$ ), utilizando-se como fonte $\mathrm{KH}_{2} \mathrm{PO}_{4}$. Os teores de $\mathrm{K}$ do substrato foram elevados para $120 \mathrm{mg} \mathrm{kg}^{-1}$, utilizando-se, como fonte, $\mathrm{KH}_{2} \mathrm{PO}_{4} \mathrm{e} \mathrm{KCl}$

\section{Inoculação e transplantio}

O inóculo utilizado foi composto de uma mistura de solo, raízes colonizadas e esporos dos FMAs. Foram utilizados $50 \mathrm{~cm}^{3}$ do inóculo de cada espécie utilizada (G. clarum, G. macrocarpum e inóculo nativo) na região, 2 a $3 \mathrm{~cm}$ abaixo da superfície do solo, em vasos de plástico com $3 \mathrm{dm}^{3}$ de capacidade.

Sementes de mamão, cultivar Improved Sunrise Solo Line $72 / 12$, foram esterilizadas superficialmente com uma solução de $0,5 \%$ de hipoclorito de sódio, por 10 minutos, e colocadas para germinar em bandejas de plástico contendo o substrato, durante quatro semanas. Após este período, três plântulas de mamão foram transferidas para os vasos.

Os compostos fenólicos foram adicionados ao substrato imediatamente após o transplantio e inoculação. Foram adicionados os seguintes compostos: rutina e quercetina, utilizando-se uma solução contendo $4 \mathrm{mg} \mathrm{L}^{-1}$, sendo adicionados $280 \mathrm{~mL}$ da solução por vaso

Os vasos foram colocados em casa de vegetação no campus da Universidade Estadual do Norte Fluminense, Campos dos Goytacazes, RJ.

\section{Delineamento experimental}

O delineamento experimental foi um fatorial de $4 \times 3 \times 2$, sendo: três tratamentos com fungo ( $G$. clarum G. macrocarpum e inóculo nativo) e testemunha nãoinfectada; dois compostos fenólicos (rutina e quercetina) e um controle; e duas doses de $\mathrm{P}$ ( 0 e $\left.50 \mathrm{mg} \mathrm{kg}^{-1}\right)$. Os tratamentos foram dispostos em blocos casualizados, com quatro repetições.

\section{Variáveis analisadas}

A avaliação do desenvolvimento das plantas foi realizada 90 dias após transplantio; nessa ocasião, avaliaramse a matéria seca da parte aérea, o conteúdo de $\mathrm{P}$, e a porcentagem de colonização micorrízica. A determinação da porcentagem de colonização micorrízica foi efetuada pelo método da interseção em placas de Petri reticuladas (Giovannetti \& Mosse, 1980), após a coloração das raízes em azul de metila (Grace \& Stribley, 1991).

O conteúdo de $\mathrm{P}$ na parte aérea foi determinado depois que as amostras foram secadas em estufa de ventilação forçada, a uma temperatura de $75^{\circ} \mathrm{C}$, por aproximadamente 48 horas (Malavolta et al., 1989). Após secadas, as amostras foram moídas em moinho tipo Willey, passadas em peneira de 20 meshes e armazenadas em frascos hermeticamente fechados. Após submeter o material vegetal à oxidação pela digestão sulfúrica, determinou-se o $\mathrm{P}$ por colorimetria, pelo método do molibdato (Malavolta et al., 1989).

\section{Análise estatística}

Foi feita a análise de variância e a comparação de médias pelo teste de Tukey a $5 \%$ de probabilidade, de todos os dados de matéria seca da parte aérea e conteúdo de $\mathrm{P}$ na parte aérea, pelo programa computacional SAEG (UFV, Viçosa)

\section{RESULTADOS E DISCUSSÃO}

A inoculação de G. clarum, na dose $0 \mathrm{mg} \mathrm{kg}^{-1}$ de $\mathrm{P}$, proporcionou aumentos significativos na produção de matéria seca e nos conteúdos de $\mathrm{P}$ da parte aérea, independentemente da adição, ou não, dos compostos rutina ou quercetina (Tabelas 1 e 2). No caso da espécie G. macrocarpum, estes aumentos só foram observados no tratamento onde se adicionou rutina, o que indica que este composto aumentou a eficiência desta espécie fúngica (Tabela 3 ). Entretanto, o aumento da colonização micorrízica nem sempre correlaciona-se com aumento do crescimento do hospedeiro. Diversos trabalhos demonstram a ocorrência de uma grande variabilidade quanto à capacidade do fungo em colonizar as raízes do hospedeiro (infectividade) e promover o crescimento vegetal (eficiência simbiótica) (Hayman \& Tavares, 1985; Haas \& Krikun, 1985, citados por Soares, 1994; 
TABELA 1. Produção de matéria seca da parte aérea $\left(\mathrm{g}_{\text {planta }}{ }^{-1}\right)$ do mamoeiro, 90 dias após plantio ${ }^{1}$.

\begin{tabular}{|c|c|c|c|c|c|c|}
\hline \multirow[t]{3}{*}{ Fungos } & \multicolumn{6}{|c|}{ Doses de $\mathrm{P}\left(\mathrm{mg} \mathrm{kg}^{-1}\right)$} \\
\hline & \multicolumn{3}{|c|}{0} & \multicolumn{3}{|c|}{50} \\
\hline & $\begin{array}{c}\text { Sem } \\
\text { composto }\end{array}$ & Rutina & Quercetina & Sem composto & Rutina & Quercetina \\
\hline Controle & $0,42 \mathrm{Ab}$ & $0,61 \mathrm{Ac}$ & $0,51 \mathrm{Ab}$ & $4,24 \mathrm{Aa}$ & $5,08 \mathrm{Aa}$ & $5,01 \mathrm{Aa}$ \\
\hline Fungo nativo & $0,31 \mathrm{Ab}$ & $0,56 \mathrm{Ac}$ & $0,57 \mathrm{Ab}$ & $3,93 \mathrm{Aa}$ & 4,52Aab & 4,30Aab \\
\hline G. clarum & $4,75 \mathrm{Aa}$ & $5,81 \mathrm{Aa}$ & $5,87 \mathrm{Aa}$ & 3,98Aa & $3,48 \mathrm{Abc}$ & $3,49 \mathrm{Ab}$ \\
\hline G. macrocarpum & $0,56 \mathrm{Bb}$ & $4,60 \mathrm{Ab}$ & $1,27 \mathrm{Bb}$ & $3,20 \mathrm{Aa}$ & $2,45 \mathrm{Ac}$ & $2,14 \mathrm{Ac}$ \\
\hline
\end{tabular}

${ }_{1}^{1}$ Médias seguidas pela mesma letra, minúscula na coluna e maiúscula na linha, não diferem entre si pelo teste de Tukey $(\mathrm{P}<0,05)$.

TABELA 2. Conteúdo de fósforo $\left(\mathrm{mg} \mathrm{planta}^{-1}\right)$ na parte aérea do mamoeiro, 90 dias após plantio ${ }^{1}$.

\begin{tabular}{|c|c|c|c|c|c|c|}
\hline \multirow[t]{3}{*}{ Fungos } & \multicolumn{6}{|c|}{ Doses de P $\left(\mathrm{mg} \mathrm{kg}^{-1}\right)$} \\
\hline & \multicolumn{3}{|c|}{0} & \multicolumn{3}{|c|}{50} \\
\hline & Sem composto & Rutina & Quercetina & Sem composto & Rutina & Quercetina \\
\hline Controle & $0,29 \mathrm{Ab}$ & $0,35 \mathrm{Ab}$ & $0,22 \mathrm{Ab}$ & $4,04 \mathrm{Abc}$ & $3,10 \mathrm{Ac}$ & $4,12 \mathrm{Ab}$ \\
\hline Fungo nativo & $0,28 \mathrm{Bb}$ & $1,10 \mathrm{Ab}$ & $0,42 \mathrm{Ab}$ & $3,36 \mathrm{Ac}$ & $4,50 \mathrm{Abc}$ & $4,12 \mathrm{Ab}$ \\
\hline G. clarum & 4,03Aa & 4,67Aa & 5,54Aa & $6,54 \mathrm{Aa}$ & 7,07Aa & $7,15 \mathrm{Aa}$ \\
\hline G. macrocarpum & $0,89 \mathrm{Bb}$ & $5,32 \mathrm{Aa}$ & $0,57 \mathrm{Bb}$ & $5,16 \mathrm{Aab}$ & 5,56Aab & $4,99 \mathrm{Ab}$ \\
\hline
\end{tabular}

${ }^{1}$ Médias seguidas pela mesma letra, minúscula na coluna e maiúscula na linha, não diferem entre si pelo teste de Tukey $(\mathrm{P}<0,05)$.

TABELA 3. Porcentagem do sistema radicular do mamoeiro colonizado pelos fungos micorrízicos arbusculares, 90 dias após plantio.

\begin{tabular}{lccccccc}
\hline Fungos & \multicolumn{7}{c}{ Doses de $\mathrm{P}\left(\mathrm{mg} \mathrm{kg}^{-1}\right)$} \\
\cline { 2 - 4 } \cline { 6 - 8 } \cline { 6 - 8 } & Sem composto & Rutina & Quercetina & & Sem composto & Rutina & Quercetina \\
\hline Controle & 0 & 0 & 0 & & 0 & 0 & 0 \\
Fungo nativo & 36 & 34 & 20 & & 35 & 77 & 66 \\
G. clanim & 58 & 60 & 58 & & 52 & 60 & 57 \\
G. macrocarpum & 36 & 49 & 34 & & 53 & 55 & 48 \\
\hline
\end{tabular}

Jaizme-Vega \& Azcón, 1995). Normalmente, a eficiência micorrízica está relacionada com a quantidade de micélio externo formado no solo. Certos fungos podem possuir grande capacidade de colonizar o hospedeiro, mas a proporção de hifas externas (estruturas que permitem a maior absorção de nutrientes) varia muito entre as espécies de FMAs (Marschner \& Dell, 1994). A eficiência micorrízica também depende de outros fatores. Algumas associações fungohospedeiro, dependendo do genótipo da planta, da espécie e ecótipo do fungo e da disponibilidade de $\mathrm{P}$ no solo, podem proporcionar apenas um baixo crescimento vegetal, chegando a atingir uma condição de parasitismo, onde o balanço energético se torna desfavorável à planta (Marschner \& Dell, 1994).

Schubert (1988) sugeriu que a eficiência da inoculação depende da presença de espécies de FMAs selecionadas. No presente trabalho, os fungos nativos, mesmo contendo a presença das espécies G. clarum e G. macrocarpum, mostraram possuir baixa eficiência simbiótica. Esta baixa eficiência do inóculo nativo pode estar relacionada à 
competitividade entre as diferentes espécies, ou ainda a maior capacidade de uma espécie micorrízica não-efetiva de colonizar preferencialmente o sistema radicular do hospedeiro.

Jaizme-Vega \& Azcón (1991) obtiveram, no tocante ao abacaxizeiro micropropagado, valores de $80 \%$ a $100 \%$ de sobrevivência, dependendo da espécie do fungo utilizada, comparados a $40 \%$ de sobrevivência das mudas não-micorrizadas. Estes mesmos autores demonstraram que a produção de biomassa aumentou em $142 \%$ e $148 \%$ com a inoculação de G. fasciculatum e G. mosseae, respectivamente, e que o conteúdo de NPK na parte aérea das mudas aumentou em $152 \%, 241 \%, 210 \%$ com o uso de $G$. fasciculatum e $270 \%, 123 \%$ e $192 \%$ com o uso de $G$. mosseae.

Num estudo mais recente, Jaizme-Vega \& Azcón (1995) obtiveram um aumento de $322 \%$ e $294 \%$ de matéria seca da parte aérea para o abacaxizeiro infectado com G. mosseae e G. fasciculatum, respectivamente, e com relação ao mamoeiro a inoculação com as mesmas espécies de FMAs também aumentou a produção de matéria seca e os conteúdos de $\mathrm{N}$, $\mathrm{P}$ e $\mathrm{K}$ da parte aérea das mudas. Tanto para o abacaxizeiro como para o mamoeiro, a inoculação das espécies acima referidas foi mais eficiente do que a inoculação dos fungos nativos.

Silva \& Siqueira (1991) observaram um aumento da matéria seca de $87 \%$ para mudas de mamoeiro infectadas com G. clarum, G. macrocarpum, $S$. heterogama e mistura de fungos (constituída de seis espécies de fungos).

Os resultados demonstraram que apenas a rutina foi capaz de proporcionar aumento na eficiência da espécie G. macrocarpum em promover o aumento no crescimento e absorção de nutrientes do mamoeiro (Tabelas 1 e 2), e que não houve influência deste composto sobre o G. clarum e o inóculo nativo.

Samarão (1998), estudando o efeito da rutina sobre a efetividade da espécie G. clarum em mudas de bananeira e goiabeira, observou que a rutina não influenciou significativamente a eficiência deste fungo, pelo fato de a espécie ter sido altamente eficiente em colonizar as raízes das plantas hospedeiras e não necessitar de efeito estimulatório da rutina.

Os compostos fenólicos têm um papel importante no controle da expressão de diferentes genes da planta e na interação simbiótica ou patogênica planta-microrganismo (Volpin et al., 1994; Peters \& Verna, 1990). Os efeitos dos compostos fenólicos nas interações simbióticas rizóbio-planta são bem estabelecidos (Lynn \& Chang, 1990; Peters \& Verna, 1990). Diversos estudos têm demonstrado que compostos fenólicos, especialmente do grupo dos flavonóides - os quais são produzidos pelas raízes como resposta de defesa à presença de patógenos - tendem a estimular o desenvolvimento de diversas espécies de FMAs (Elias \& Safir, 1987; Tsai \& Phillips, 1991; Bécard et al., 1992; Baptista \& Siqueira, 1994). Siqueira et al. (1991) sugerem que os estudos com compostos fenólicos abrem novas perspectivas para a utilização de FMAs, aumentando a colonização e maximizando os benefícios que estes fungos proporcionam às plantas hospedeiras. Estes mesmos autores referem-se à utilização de compostos fenólicos no sentido de estimular a população nativa de FMA, e destacam o isoflavonóide formononetina como o mais ativo. Entretanto, os compostos fenólicos utilizados no presente estudo não estimularam as populações nativas de FMAs

Com aumento da dose de $\mathrm{P}$ adicionada ao substrato, observou-se que as espécies de FMAs não promoveram aumentos significativos sobre a produção de matéria seca e conteúdos de $\mathrm{P}$ da parte aérea (Tabelas 1 e 2). O crescimento de plantas infectadas com FMAs é influenciado pelos níveis de $\mathrm{P}$ do solo. Em condições subótimas de $\mathrm{P}$, a taxa de colonização é geralmente elevada, e a planta hospedeira é beneficiada com esta associação; porém, em níveis elevados de $\mathrm{P}$, a associação micorrízica pode exercer efeito depressivo à planta hospedeira (Saggin Júnior \& Siqueira, 1995), podendo tornar-se parasítica (Bethlenfalvay et al., 1983). Veen et al. (1989) demonstraram que os FMAs podem consumir cerca de $30 \%$ do $\mathrm{C}$ fotoassimilado pela planta hospedeira, o que poderia ocasionar uma supressão no crescimento do hospedeiro. Diversos autores (Marschner \& Dell, 1994; Tawaraya et al., 1994; Chandrashekara et al., 1995) constataram que os benefícios dos FMAs para o crescimento das plantas diminui quando a disponibilidade de $\mathrm{P}$ é alta. Em um experimento comparando o efeito do suprimento de $\mathrm{P}$ no crescimento de mudas de bananeira infectadas com FMAs, Lin \& Fox (1992) demostraram que a colonização por FMAs 
diminuiu com o aumento da disponibilidade de $\mathrm{P}$ no solo. Altas aplicações de $\mathrm{P}$ ao solo diminuem a formação dos FMAs, e este efeito é atribuído ao aumento de P na planta (Menge et al., 1978; Schwab et al., 1983; Amijee et al., 1989). Os mecanismos que regulam a colonização do fungo em função dos níveis de $\mathrm{P}$ ainda não estão bem definidos. Alguns estudos têm demonstrado que níveis elevados de $\mathrm{P}$ causam um decréscimo na concentração de carboidratos, ácidos carboxílicos e aminoácidos nos exsudatos das raízes, e um decréscimo na concentração de ácidos carboxílicos nos extratos das raízes (Schwab et al., 1983). Graham et al. (1981) observaram que o aumento na exsudação de carboidratos e aminoácidos pelas raízes está associado a um aumento da permeabilidade da membrana das raízes com deficiência de $\mathrm{P}$, e não a mudanças no conteúdo de carboidratos e aminoácidos nas raízes. Estes autores sugerem que condições de deficiência de $\mathrm{P}$ causam aumento na permeabilidade da membrana da raiz, que leva à perda de metabólitos que favorecem a germinação e crescimento do fungo nos períodos de pré e pós-infecção. Entretanto, em condições de alta disponibilidade de $\mathrm{P}$ ocorre uma diminuição da permeabilidade da membrana citoplasmática da raiz, diminuindo a exsudação de carboidratos e aminoácidos

\section{CONCLUSÕES}

1. Os fungos Glomus clarum e G. macrocarpum (acrescido de rutina) proporcionam aumentos significativos no crescimento e no conteúdo de $\mathrm{P}$ das mudas de mamoeiro, apenas na menor dose de $\mathrm{P}$.

2. A rutina aumenta a eficiência da espécie G. macrocarpum, o que resulta em incremento no crescimento e na absorção de nutrientes do mamoeiro; nenhuma influência deste composto é observada sobre o G. clarum e o inóculo nativo.

\section{AGRADECIMENTOS}

À Dra. Rosilaine Carrenho e Dra. Sandra Truffen, do Instituto Botânico de São Paulo, Setor de Micologia, pela identificação das espécies nativas de FMAs.

\section{REFERÊNCIAS}

AMIJEE, F.; TINKER, P.B.; STRIBLEY, D.P. The development of endomycorrhizal root system. VII. A detailed study of effects of soil phosphorus on colonization. New Phytologist, Cambridge, GrãBretanha, v.111, p.435-446, 1989.

BAPTISTA, M.J.; SIQUEIRA, J.O. Efeito de flavonóides na germinação de esporos e no crescimento assimbiótico do fungo micorrízico arbuscular Gigaspora gigantea. Revista Brasileira de Fisiologia Vegetal, Londrina, v.6, p.127-134, 1994.

BECÁRD, G.; DOUDS, D.D.; PFEFFER, P.E. Extensive in vitro growth of vesicular-arbuscular mycorrhizal fungi in the presence of $\mathrm{CO}_{2}$ and flavonoids. Applied and Environmental Microbiology, Washington, v.58, p.821-825, 1992.

BETHLENFALVAY, G.J.; BAYNE, H.G.; PACOVSKY, R.S. Parasitic and mutualistic associations between a mycorrhizal fungus and soybean: the effect of phosphorus on the host plant-endophyte interactions. Journal of Plant Physiology, Stuttgart, v.57, p.543-548, 1983.

CHANDRASHEKARA, C.P.; PATIL, V.C.; SREENIVASA, M.N. VA-mycorrhiza mediated P effect on growth and yield of sunflower (Helianthus annuus L.) at different P levels. Plant and Soil, Dordrecht, v.176, p.325-328, 1995.

ELIAS, K.S.; SAFIR, G.R. Hyphal elongation of Glomus fasciculatus in response to root exudates. Applied and Environmental Microbiology, Washington, v.53, p.1928-1933, 1987.

GIOVANNETTI, M.; MOSSE, B. An evaluation of techniques for measuring vesicular-arbuscular mycorrhizal infection in roots. New Phytologist, Cambridge, Grã-Bretanha, v.84, p.489-500, 1980.

GRACE, C.; STRIBLEY,D.P. A safer procedure for routine staining of vesicular-arbuscular mycorrhizal fungi. Mycological Research, Cambridge, Grã-Bretanha, v.95, p.1160-1162, 1991.

GRAHAM, J.H.; LEONARD, R.T.; MENGE, J.A. Membrane-mediated decrease in root exudation responsible for phosphorous inhibition of vesiculararbuscular mycorrhiza formation. Journal of Plant Physiology, Stuttgart, v.68, p.548-552, 1981.

HAYMAN, D.S.; TAVARES, M. Plant growth responses to vesicular-arbuscular mycorrhiza. XI. Influence of 
soil $\mathrm{pH}$ on the symbiotic efficiency of different endophytes. New Phytologist, Cambridge, GrãBretanha, v.100, p.367-377, 1985.

JAIZME-VEGA, M.C.; AZCÓN, R. Effect of vesiculararbuscular mycorrhizal fungi on pineapple [Ananas comosus (L.) Merr.] in the Canary Islands. Fruits, Paris, v.46, p.47-50, 1991.

JAIZME-VEGA, M.C.; AZCÓN, R. Responses of some tropical and subtropical cultures to endomycorrhizal fungi. Mycorrhiza, Berlin, v.5, p.213-217, 1995

LIN, M.L.; FOX, R.L. The agronomic effectiveness of rock phosphate and superphosphate for banana Fertilizer Research, Dordrecht, v.31, p.131-135, 1992

LYNN, D.G.; CHANG, M. Phenolic signals in cohabitation implications for plant development. Annual Review of Plant Physiology and Plant Molecular Biology, Palo Alto, v.41, p.497-526, 1990.

MALAVOLTA, E.; VITTI, G.C.; OLIVEIRA, S.A. Avaliação do estado nutricional das plantas. Piracicaba : Associação Brasileira para Pesquisa da Potassa e do Fosfato, 1989. 201p.

MARSCHNER, H.; DELL, B. Nutrient uptake in mycorrhizal symbiosis. Plant and Soil, Dordrecht, v.159, p.89-102, 1994.

MENGE, J.A.; STEIRLE, D.; BAGYARAJ, D.J.; JOHNSON, E.L.V.; LEONARDI, R.T. Phosphorus concentration in plants responsible for inhibition of mycorrhizal infection. New Phytologist, Cambridge, Grã-Bretanha, v.80, p.575-578, 1978.

PETERS, N.K.; VERNA, P.S. Phenolic compounds as regulators of gene expression in plant-microbe interactions. Molecular Plant-Microbe Interactions, Saint Paul, v.3, p.4-8, 1990

SAGGIN JÚNIOR, J.P.; SIQUEIRA, J.O. Avaliação da eficiência simbiótica de fungos endomicorrízicos no cafeeiro. Revista Brasileira de Ciência do Solo, Campinas, v.19, p.221-228, 1995.

SAMARÃO, S.S. Influência de fungos micorrízicos arbusculares na produção de mudas de bananeira (Musa spp.) e goiabeira (Psidium guajava L.). Campos dos Goytacazes : Universidade Estadual do Norte Fluminense, 1998. 61p. Dissertação de Mestrado.
SCHUBERT, A. Growth and root colonization of grapevines inoculated with different mycorrhizal endophytes. Horticultural Science, Calcutta, v.23, p.302-303, 1988.

SCHUBERT, A.; MAZZITELLI, M.; ARIUSSO, O.; EYNARD, I. Effects of vesicular-arbuscular mycorrizal fungi on micropropagated grapevines: Influence of endophyte strain, P fertilization, and growth medium. Vitis, Sielbeldingen, v.29, p.5-13, 1990

SCHWAB, S.M.; MENGE, J.A.; LEONARD, R.T. Comparison of stages of vesicular-arbuscular mycorrhiza formation in sudan-grass grown at two levels of phosphorus nutrition. American Journal of Botany, Columbus, v.70, p.1225-1232, 1983.

SILVA, L.F.C.; SIQUEIRA, J.O. Crescimento e teores de nutrientes de mudas de abacateiro, mangueira e mamoeiro sob a influência de diferentes espécies de fungos micorrízicos vesículo-arbusculares. Revista Brasileira de Ciência do Solo, Campinas, v.15, p.283288,1991

SIQUEIRA, J.O.; NAIR, M.G.; HAMMERSCHIMIDT, R.; SAFIR, G.R. Significance of phenolic compounds in plant-soil microbial systems. Critical Reviews in Plant Sciences, Boca Raton, v.10, p.63-121, 1991.

SOARES, I. Associação micorrízica na cultura do maracujá. In: SÃO JOSÉ, A.R. (Ed.). Maracujá: produção e mercado. Vitória da Conquista : Universidade Estadual Sudoeste da Bahia, 1994. p.91-98.

TAWARAYA, K.; SASAI, K.; WAGATSUMA, T. Effect of phosphorus application on the contents of amino acids and reducing sugars in the rhizosphere and VA mycorrhizal infection of white clover. Soil Science and Plant Nutrition, Tokyo, v.40, p.539-543, 1994.

TSAI, S.N.; PHILLIPS, D.A. Flavonoids released naturally from alfalfa promote development of symbiotic Glomus spores "in vitro". Applied and Environmental Microbiology, Washington, v.57, p.1485-1488, 1991

VEEN, J.A. van; MERCKX, R.; GEIJ, S.C. van de. Plant and soil related controls of the flow of carbon from roots through the soil microbial biomass. Plant and Soil, Dordrecht, v.115, p.179-188, 1989.

VOLPIN, H.; ELKIND, Y.; OKON, Y.; KAPULNIK, Y. A vesicular arbuscular mycorrhizal fungus (Glomus intradix) induces a defense response in alfalfa roots. Plant Physiology, Rockville, v.104, p.683-689, 1994. 